\title{
The protective effect of Vitamin A Palmitate Eye Gel on ocular surface during general anesthesia surgery: a randomized clinical trial
}

\section{Siyuan Li}

Beijing Institute of Ophthalmology

\section{Guiyu Lei}

department of anesthesiology, beijing tongren hospital

Ying Liu

operating room, beijing tongren hospital

Lei Tian

Beijing Institute of Ophthalmology

Ying Jie ( $\nabla$ jie_yingcn@aliyun.com )

Beijing Tongren HospitalDepartment of Ophthalmology: Beijing Tongren Eye Center https://orcid.org/0000-0003-2649-8241

\section{Guyan Wang}

deparment of anesthesiology, beijing tongren hospital

\section{Research}

Keywords: general anesthesia, ocular surface, postoperative dry eye, Vitamin A Palmitate Eye Gel, protection.

Posted Date: February 18th, 2022

DOI: https://doi.org/10.21203/rs.3.rs-1302316/v1

License: (c) (1) This work is licensed under a Creative Commons Attribution 4.0 International License. Read Full License 


\section{Abstract}

Background: General anesthesia was associated with tear production decreased, usually accompanied by ophthalmological complications, such as dry eye and corneal injuries. Patients suffering from such conditions developed symptoms such as conjunctival injection, eye pain, and tearing. Protective measures including taping eyelid closed and applying lubricating tears during general anesthesia have long been advocated to reduce the incidence of corneal abrasion. The study aimed to investigate the change of tear production associated with general anesthesia, and the protective effect of Vitamin A Palmitate Eye Gel on ocular surface during general anesthesia, including the subjective symptoms and objective signs.

Methods: The study was designed as a double-blind randomized clinical trial. Each participant who was ready for non-ophthalmic general anesthesia surgery randomly received Vitamin A Palmitate Eye Gel and taping for one eye (Group A, $n=60$ ), and taping alone for the other eye (Group B, $n=60$ ) though random number table. Symptom assessment in dry eye (SANDE) score, tear film break-up time (TBUT), corneal fluorescein staining (CFS) score and Schirmer tear test I (STT-1) were assessed under hand-held slit lamp before anesthesia $\left(T_{0}\right), 0.5 \mathrm{~h}$ postoperative $\left(T_{1}\right)$ and $24 \mathrm{~h}$ postoperative $\left(T_{2}\right)$.

Results: At $0.5 \mathrm{~h}$ postoperative, both groups demonstrated increase in CFS score ( $P<0.05$ in Group A and $P<0.01$ in Group B), and the participants in Group A showed numerically less corneal abrasion than that of in Group B. STT-1 observed in Group A was statistically increased $(P<0.05)$, while that of in Group B was statistically decreased $(P<0.001)$. The changes between two groups were statistically significant $(P<0.001)$. At 24h postoperative, both CFS score and STT-1 almost returned to baseline level in two groups. SANDE score and TBUT showed little change at $0.5 \mathrm{~h}$ and $24 \mathrm{~h}$ postoperative in two groups $(P>0.05)$.

Conclusion: Vitamin A Palmitate Eye Gel were effective in ocular surface protection and aqueous supplementation during general anesthesia.

Trial registration: The study was registered with the Chinese Clinical Trial Registry (ChiCTR2100052140). Registered 20 October,2021. http://www.chictr.org.cn/showproj.aspx?proj=135706

Trial registration: ISRCTN, ISRCTN12345678., http://www.isrctn.com/ISRCTN12345678

\section{Introduction}

General anesthesia surgery was often followed by ophthalmological complications, such as dry eye disease (DED) and corneal injuries, which include corneal abrasion, recurrent corneal erosion, and exposure keratitis. Studies have shown that the incidence of postoperative corneal abrasion can reach $59 \%$, if no protective measures were taken during surgery ${ }^{[1]}$. Such side-effects can cause discomfort 
symptoms such as conjunctival injection, eye pain, and tearing after the operation, which not only affect the patient's recovery, but may also cause serious ocular complications such as corneal ulcers and vision loss.

Therefore, ocular surface protection in general anesthesia is a problem that both ophthalmologists and anesthesiologists are concerned about. At present, the most commonly used protective measure is to tape the eyelids closed, however, some patients will complain of dry eyes and astringent eyes after surgery, which may be related to the suppression of lacrimal secretion by some anesthetic auxiliary drugs $^{[2]}$.

Ziyang ${ }^{\circledR}$ Vitamin A Palmitate Eye Gel (Shenyang Xingqi Pharmaceutical Co.,Ltd, Shenyang, China) is a medicine mainly used to repair corneal epithelial cells after ocular surface damage, which is commonly used for adjuvant treatment of dry eyes, meibomian gland dysfunction, and exposure keratitis. The vitamin A component is a necessary condition for the normal differentiation of epithelial cells, and the palmitate component can effectively replenish tear film lipid layer and reduce tear evaporation. The matrix is carbomer with high viscosity, which can enhance the protection of gel by physical lubrication.

The study aimed to investigate the change of tear production associated with general anesthesia, and the protective effect of Vitamin A Palmitate Eye Gel on ocular surface during general anesthesia, including the subjective symptoms and objective signs.

\section{Methods}

Study design

This single centered, randomized, double-blind study was performed in Beijing Tongren Hospital, which was approved by the Medical Ethics Committee of Beijing Tongren Hospital, Capital Medical University (No. TRECKY2021-137), and was registered with the Chinese Clinical Trial Registry (ChiCTR2100052140). 60 participants ( 120 eyes ) for non-ophthalmic general anesthesia surgery in November 2021 were included in this study and all participants were fully explained and signed the informed consent. The anesthesiologist is a specialized doctor and responsible for the grouping. Each participant randomly received Vitamin A Palmitate Eye Gel and taping for one eye (Group A, $n=60$ ), and taping alone for the other eye (Group $B, n=60$ ) through random number table. The ophthalmologist is responsible for ocular examination and evaluation. The specific medication eyes were blind to both ophthalmologist and participants involved in the study.

\section{Participants}

Inclusion criteria were the followings: $₫$ aged $\geq 18$ years; 『patients undergoing non-ophthalmological surgery under general anesthesia of $>2$ hours in the supine position; 咕e scope of surgical disinfection does not involve periocular skin; 『voluntarily participated in this study and could cooperate with the whole research process. 
Exclusion criteria were the followings: 『with active ocular diseases such as keratitis; 『with ocular surface

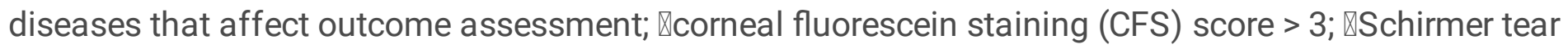
test I (STT-1) $<5 \mathrm{~mm}$ and tear film break-up time (TBUT) $<5 \mathrm{~s}$; खRobertson consciousness score $<4$, unable to cooperate with the inspection; $₫$ Allergic person.

\section{Study process}

All patients received general anesthesia with laryngeal mask or tracheal intubation. Anesthesia was induced with sufentanil $(0.1-0.3 \mu \mathrm{g} / \mathrm{kg})$, propofol $(1-2 \mathrm{mg} / \mathrm{kg})$, and rocuronium $(0.6 \mathrm{mg} / \mathrm{kg})$. Then the anesthesiologist applied the eye gel to the conjunctival sac of the lower eyelid of the experimental eye before taped both eyelids closed horizontally. Anesthesia was maintained by continuous infusion of propofol and remifentanil and meanwhile sevoflurane alone or propofol alone or combined decided by different anesthesiologists. During the operation, electrocardiogram, pulse oximetry, noninvasive arterial blood pressure, and end-tidal carbon dioxide concentration were monitored. Sedation level was kept within the range of $40-60$ under bispectral index (BIS) monitor. The fluctuation of mean atrial pressure(MAP)was maintained within $20 \%$ of the preoperative values of the patients. All patients received i.v lactated Ringer's solution at a rate of $5-7 \mathrm{ml} / \mathrm{kg}$. Atropine and neostigmine was given at the discretion of the anesthetist at the end of surgery but prior to extubation. The anesthesiologist removed the tape, wiped the residual gel composition around the test eye, and push the subject into the anesthesia recovery room.

\section{Measures and outcomes}

The eyes were examined under hand-held slit lamp before anesthesia (recorded as $T_{0}$ ), 0.5 postoperative (measured in the anesthesia recovery room, recorded as $\mathrm{T}_{1}$ ) and $24 \mathrm{~h}$ postoperative (recorded as $\mathrm{T}_{2}$ ). Examination in all participants were arranged in following order to avoid unexpected corneal abrasion.

Subjective symptom score: Symptom Assessment in Dry Eye (SANDE) questionnaire was used for evaluating participants' subjective symptom. The questionnaire was composed of two parts based on the visual analogue scale, which could quantitatively indicate the frequency and severity of ocular surface diseases feasibly ${ }^{[3]}$.

TBUT: After moistening the fluorescein sodium staining strip (Jingming New Technology Development Co., Ltd., Tianjin, China) with chloramphenicol eye drops, smeared on the nasal side, central side and temporal side of the lower eyelid margin. Asked the participants to close their eyes for 3 seconds and open. Observe the tear film with a cobalt blue light and recorded the time from the last blink to the occurrence of tear film rupture. The average value was taken for three times.

CFS score: Corneal abrasion was measured using cobalt blue light following TBUT evaluation. The denuded area of corneal epithelium was stained green whereas the normal surface was left unaltered. According to the guidelines of the National Institutes of Ophthalmology / Industry Working Group, five 
corneal regions ( middle, nasal, temporal, upper and lower ) were scored, with 0-3 points in each region, ranging from 0 to 15 points ${ }^{[4]}$.

Tear production evaluation (STT-1): It was the primary outcomes in this study. In the absence of ocular surface anesthesia, placed the filter strip (Jingming New Technology Development Co., Ltd., Tianjin, China) in the temporal inferior conjunctival sac, and asked the participants to close the eyes. Both eyes were evaluated simultaneously. Took the filter strip out after 5 minutes and read the wet length as the result.

Safety outcomes: visual acuity, anterior segment and facial skin were observed at each timepoint.

\section{Statistical analysis}

Statistical analyses of the outcomes were conducted by SPSS22.0 (IBM Corp, Somers, NY). Median and interquartile intervals were used to describe data of ranked data (SANDE score and CFS score) and abnormal distribution (TBUT and STT-1).

The Mann-Whitney $U$ test was conducted to analyze the changes from baseline in the outcome measures, and the Wilcoxon rank-sum test was used for comparisons between Group A and Group B. All the hypotheses were tested as two-sided, and the difference was statistically significant with $P<0.05$.

The power for the STT-1 change was calculated based on a two sided t-test with a significance level of $5 \%$. Using a power of $90 \%$ to detect a difference between the two group in STT-1, given that the probabilities that STT-1 would decrease to $3.6 \mathrm{~mm} / 5 \mathrm{mins}$ in Group A and $4.3 \mathrm{~mm} / 5 \mathrm{mins}$ in Group B with a standard deviation of 1.1, the minimum sample size required to detect a statistical difference was 52 subjects in each group. Assuming the drop-out rate is $10 \%$, it was appropriate to enroll 60 eyes in each group ${ }^{[5]}$.

\section{Results}

A total of 60 participants (120 eyes) were included in this study, while 58 participants (116 eyes) completed the entire trial process. Figure 1 shows the CONSORT flow diagram, including information about study exclusion and the sample size for analysis. Table 1 summarizes the baseline characteristics of participants in this study. The baseline levels (recorded as $T_{0}$ ) were listed in Table 2. Since the study was designed as self-controlled, the data were well-matched in terms of baseline characteristics between two groups.

Compared with baseline SANDE scores, there were similarly little change at $0.5 \mathrm{~h}$ and $24 \mathrm{~h}$ postoperative in Group A (change in scores $=14.31$ and 13.36, respectively) $(P>0.05)$ and in Group $B$ (change in scores $=$ 13.01 and 13.43 , respectively) ( $P>0.05$ ). There was no difference in the change of the assessment between Group A and Group B at $0.5 \mathrm{~h}$ and $24 \mathrm{~h}$ postoperative $(P>0.05)$. 
In both Group A and Group B, TBUT decreased significantly from baseline level at 0.5 h postoperative (change in TBUT $=6.48 \mathrm{~s}$ and $6.04 \mathrm{~s}$, respectively) $(P<0.001)$. At $24 \mathrm{~h}$ postoperative, TBUT almost returned to baseline level in both Group A and Group B (change in TBUT $=8.19 \mathrm{~s}$ and $7.32 \mathrm{~s}$, respectively) $(P>0.05)$. The change trends in both groups were equivalent without statistical differences $(P>0.05)$.

At $0.5 \mathrm{~h}$ postoperative, increase in CFS score compared with baseline was statistically significant in Group A (change in scores $=1)(P<0.05)$ and in Group B (change in scores $=1)(P<0.01)$. In direct comparison, the participants in Group A showed numerically less corneal abrasion than that of in Group B. At $24 \mathrm{~h}$ postoperative, there was no statistical difference from baseline level in both Group A and Group B (change in scores $=1$ and 1 , respectively $)(P>0.05)$.

Statistically significant improvement in STT-1 was observed at $0.5 \mathrm{~h}$ postoperative in Group A (change in $\mathrm{STT}=7.5 \mathrm{~mm})(P<0.05)$. However, there was a statistically decrease in Group B (change in STT $=4 \mathrm{~mm}$ ) $(P<0.001)$. The changes between two groups were statistically significant $(P<0.001)$. At $24 \mathrm{~h}$ postoperative, STT-1 almost returned to baseline level in both Group A and Group B (change in STT = $6 \mathrm{~mm}$ and $6 \mathrm{~mm}$, respectively) ( $P>0.05)$.

Figure1. CONSORT flow diagram. 
Table 1

Characteristics of the study participants.

\begin{tabular}{|ll|}
\hline Variable & Participants \\
\hline Gender & \\
\hline Female & $33(56.9 \%)$ \\
\hline Male & $25(43.1 \%)$ \\
\hline Age (years) & $46.03 \pm 11.94$ \\
\hline BMI (kg/cm2) & $23.25 \pm 2.79$ \\
\hline Smoking & \\
\hline Yes & $8(13.8 \%)$ \\
\hline No & $50(86.2 \%)$ \\
\hline Surgery & \\
\hline thyroid surgery & $41(70.7 \%)$ \\
\hline orthopedic surgery & $9(15.5 \%)$ \\
\hline breast surgery & $7(12.1 \%)$ \\
\hline gallbladder surgery & $1(1.7 \%)$ \\
\hline Operation time (mins) & $179.05 \pm 41.30$ \\
\hline Operation position & \\
\hline supine & $58(100 \%)$ \\
\hline ASA grade & $22(37.9 \%)$ \\
\hline 1 & $36(62.1 \%)$ \\
\hline 2 & \\
\hline
\end{tabular}


Table 2

Satisfaction comparison between two groups, median $\left(\mathrm{P}_{25}, \mathrm{P}_{75}\right)$

\begin{tabular}{|c|c|c|c|c|c|}
\hline & $\begin{array}{l}T_{0} \\
(n=120)\end{array}$ & $\begin{array}{l}T_{1} \\
(n=116)\end{array}$ & $\begin{array}{l}T_{2} \\
(n=116)\end{array}$ & $P T_{0}-T_{1}$ & $P T_{0}-T_{2}$ \\
\hline \multicolumn{6}{|c|}{ SANDE score (points) } \\
\hline Group A & $13.47(4.13,20.95)$ & $14.31(4.13,20.95)$ & $13.36(4.33,20.65)$ & 0.25 & 0.61 \\
\hline Group B & $13.54(4.51,23.86)$ & $13.01(4.01,20.98)$ & $13.43(4.14,21.27)$ & 0.95 & 0.46 \\
\hline$P$ & 0.95 & 0.42 & 0.78 & & \\
\hline \multicolumn{6}{|l|}{ TBUT (s) } \\
\hline Group A & $8.56(6.00,10.29)$ & $6.48(4.94,8.31)$ & $8.19(6.59,9.49)$ & $0.0001^{\star \star \star}$ & 0.26 \\
\hline Group B & $8.25(5.64,10.54)$ & $6.04(4.60,6.79)$ & $7.32(6.00,8.61)$ & $0.0001^{\star \star *}$ & 0.07 \\
\hline$P$ & 0.64 & 0.72 & 0.55 & & \\
\hline \multicolumn{6}{|c|}{ CFS score (points) } \\
\hline Group A & $0(0,1)$ & $1(0,1)$ & $0(0,0)$ & $0.011 *$ & 0.05 \\
\hline Group B & $0(0,1)$ & $1(0,1)$ & $0(0,1)$ & $0.001^{\star *}$ & 0.57 \\
\hline$P$ & 0.50 & 0.50 & 0.25 & & \\
\hline \multicolumn{6}{|c|}{ STT-1 (mm/5mins) } \\
\hline Group A & $6(4,8)$ & $7.5(4.25,10)$ & $6(4,8)$ & $0.02^{\star}$ & 0.68 \\
\hline Group B & $6(3.25,10)$ & $4(3,5)$ & $6(4,8)$ & $0.0001^{\star \star \star}$ & 0.14 \\
\hline$P$ & 0.91 & $0.0001 * \star \star$ & 0.32 & & \\
\hline
\end{tabular}

${ }^{*} \mathrm{P}<0.05,{ }^{* \star P} \mathrm{P}<0.01, * \star * \mathrm{P}<0.001$

$T_{0}$ before general anesthesia (baseline), $T_{1}$ at postoperative $0.5 \mathrm{~h}, \mathrm{~T}_{2}$ at postoperative $24 \mathrm{~h}$

CFS = Corneal fluorescein staining; SANDE = Symptom assessment in dry eye; STT = Schirmer tear test; TBUT = Tear film break-up time

Figure 2. Hand-held slit lamp corneal fluorescein sodium-stained cobalt blue photography. This participant underwent orthopedic surgery, and the duration of anesthesia was 197 minutes. His conjunctival sac of the right eye (OD) was smeared with VA ophthalmic gel, and the adhesive tape was transversely pasted. His left eye (OS) was simply pasted with adhesive tape. There were several fluorescein dots on the cornea of the left eye at $T_{1}$ (arrow).

\section{Discussion}


In the present study, we aimed to investigate the protective effect of Vitamin A Palmitate Eye Gel on ocular surface during general anesthesia. This trial was designed as a randomized double-blind selfcontrol study, and we compared the protection effect of taping eyelid alone and combination with Vitamin A Palmitate Eye Gel on subjective symptoms and objective signs of each participant. In our study, it could be observed that in addition to CFS score increasing slightly, the STT-1 and TBUT decreased significantly, which represented that those participants suffered not only from corneal abrasions, but also from postoperative DED.

DED is a multifactorial disease of the ocular surface characterized by a loss of homeostasis of the tear film that has a vital role in providing lubrication and protection to the cornea ${ }^{[6]}$. Postoperative DED followed by general anesthesia surgery, however, has not drawn much attention from the surgeons. Such patients may not present with significant pain due to no instant corneal abrasions. Nonetheless, they would develop progressing DED in a few days or even weeks with discomfort symptoms appearing and associated signs maintaining, such as decreased STT and TBUT.

For now, reduced tear production was considered as the main pathogenic factors of postoperative DED. Tear production is controlled by the autonomic nervous system, of which the parasympathetic system dominated. There are $\mathrm{G}$ protein-coupled muscarinic receptors on the surface of lacrimal gland acinar cells and conjunctival goblet cells, which are regulated by acetylcholine (Ach). Atropine, as an anticholinergic drug often used in anesthesia induction and recovery, block the muscarinic receptors, and reduce tear secretion. Krupin et al. measured the basal tear production by standardized Schirmer strips in 20 patients and the results showed a significant decrease at 10,30 and 60 minutes following induction of the anesthesia ${ }^{[5]}$. Decreased tear production would induce the deficiency of the aqueous layer.

Recent studies showed that general anesthesia may alter tear biochemistry as well ${ }^{[7]}$. Zernii et al. showed that the development of chronic postoperative DED was accompanied by a decrease in teal film stability, which due to the decrease in total antioxidant activity of the tear ${ }^{[8]}$. And they observed that anesthesia induced changes in activity of tear antioxidant enzymes including superoxide dismutase and enzymes providing homeostasis of reduced glutathione.

Batra and Bali first paid attention to the ocular surface protection during general anesthesia in $1977^{[9]}$. Taping eyelid closed, which is most commonly used protection method, has certain limitations, such as inconvenient observation of the pupil, improper pasting or recovery stage untimely removal of the tape may cause ocular surface mechanical damage. Besides, it did not provide any ingredient supplement to the tear film.

Vitamin A, as the main component of the eye gel, plays an important role in regulating epithelial growth, cell proliferation and differentiation. It was proved that in human corneal conjunctival cells, Vitamin A upregulated secretory phospholipase $A$ group IIA genes to increase mucin 16 expression $^{[10]}$. At the meantime, numbers of population studies showed that systemic Vitamin A supplementation could improve tear quality through repopulating conjunctiva goblet cell, increasing their density and helping 
corneal re-epithelization ${ }^{[11-13]}$. Lots of clinical studies have proved that topical supplementation of Vitamin A was effective in ocular surface condition improvement ${ }^{[14]}$. A study enrolled dry eye patients who were unresponsive to conventional treatments and found that Vitamin A ointment were largely effective in reducing signs and symptoms, also promoting goblet cell proliferation by more than $70 \%{ }^{[15]}$.

The palmitate component can effectively replenish tear film lipid layer and reduce tear evaporation. The matrix is carbomer with high viscosity, which can enhance the protection of gel by physical lubrication.

In the current study, it could be seen that the STT- 1 measured $0.5 \mathrm{~h}$ postoperative in Group B was significantly decreased, but that of in Group A was increased (Fig. 2). STT is a well- standardized test which provides an estimation of basic tear secretion. The result of Group B was corresponded with the existing scientific literature on general anesthesia and tear secretion. The increase of STT-1 in Group A might have several reasons. First, mucin expression promoted by Vitamin A could help increase tear film stability further. Second, carbomer, as the matrix of Vitamin A, is kind of synthetic high molecular weight polymer of acrylic acid cross-linked to a polyalkenyl polyether, which form a liquid reservoir inside the gel after acting on the ocular surface ${ }^{[16]}$. It could release drugs slowly and permanently. In addition, gel-based artificial tear supplements could offer higher viscosity and loner retention times on the ocular surface, meanwhile, it is less sticky than oil-based formulations. Thus, the aqueous composition still maintains sufficient for tear film in Group A after surgery, reflecting in increasing STT-1 measured $0.5 \mathrm{~h}$ postoperative.

The results showed that the CFS score measured $0.5 \mathrm{~h}$ postoperative increased slightly in two groups. Referred to the incidence of corneal abrasion without ocular protection ${ }^{[9,17]}$, both eye gel and typing the eyelids could effectively make a difference. The CFS score in Group A was numerically lower than that of in Group B, which might benefit from the function of Vitamin A in maintaining the health of epithelial cells. TBUT in both groups decreased at $0.5 \mathrm{~h}$ postoperative and showed no statistical difference between two groups. TBUT was the most frequently employed test of ocular surface stability, and any factor that affected the composition of the tear film could lead to a decrease. According to the previous literatures, Vitamin A Palmitate Eye Gel was able to prevent the destruction of the tear film homeostasis aiming to the ocular surface that after ocular surgery or influenced by some eye drops ${ }^{[18,19]}$. There is currently few research using Vitamin A Palmitate Eye Gel to prevent postoperative DED.

Furthermore, it could be seen from the baseline that the preoperative STT-1 and TBUT were lower than the normal level, but only two participants showed to the doctor that they were diagnosed as DED, suggesting the characteristic of separation of symptoms and signs. Many DED patients, especially middle-aged and elderly people, perimenopausal people or visual display terminal users, although related signs were developed, would not go for medical intervention because of no obvious symptoms. It was proved that with a healthy ocular surface environment, the reduction of tear secretion caused by general anesthesia would not cause dyshomeostasis under the condition of taping eyelid closed alone ${ }^{[20]}$. However, if the patient had already suffered from ocular surface diseases such as DED or had shown ocular surface 
dyshomeostasis before the surgery, in addition to the decrease in tear secretion, it could also cause delay in lipid layer distribution and further loss of aqueous during blink.

The current study still showed certain limitations. Some participants were not totally conscious when answering questionnaire, therefore the SANDE score probably could not show their feelings accurately. Future research will investigate the protective effectiveness of Vitamin A Palmitate Eye Gel in long surgery procedures and the surgery in lateral or prone position.

\section{Conclusion}

Vitamin A Palmitate Eye Gel could provide additional protection and aqueous supplement to the ocular surface during general anesthesia, especially in patients who have suffered from ocular surface diseases such as DED.

\section{Abbreviations}

CFS

Corneal fluorescein staining

STT

Schirmer tear test I

TBUT

Tear film break-up time

SANDE

Symptom assessment in dry eye

DED

dry eye disease

\section{Declarations}

\section{Ethics approval and consent to participate}

The study was approved by the Medical Ethics Committee of Beijing Tongren Hospital, Capital Medical University (No. TRECKY2021-137). All participants were fully explained and signed the informed consent.

\section{Consent for publication}

All authors have read the manuscript, have approved the paper, and agreed to it being submitted for publication.

\section{Data Availability}

The data used to support the findings of this study are available from the corresponding author upon request. 


\section{Competing interests}

The authors declare no conflicts of interest.

\section{Funding}

This study was supported by "The Youth Beijing Scholars program" and Beijing Hospitals Authority Clinical Medicine Development of Special Funding Support, (code: ZYLX202103).

\section{Authors' contributions}

Ying Jie and Guyan Wang were the main persons in charge of this clinical trial. Guiyu Lei were responsible for participants enrolled, grouping and anesthesia. Siyuan Li and Lei Tian were responsible for eye examination. Ying Liu was responsible for nursing. Siyuan Li wrote the main manuscript text. All authors reviewed the manuscript.

\section{Acknowledgments}

Not applicable.

\section{References}

1. Justin MPappA, Vernau GA, Aden CT, Fitzgerald JK, Kraus BM. GP, et al. Perioperative Corneal Abrasions After Nonocular Surgery: A Systematic Review Cornea. 2019;38(7):927-32.

2. Krupin T, Cross DA, Becker B. Decreased Basal Tear Production Associated With General Anesthesia. Arch Ophthalmol. 1977;95(1):107.

3. Schaumberg DA, Gulati A, Mathers WD, Clinch T, Lemp MA, Nelson JD, et al. Development and Validation of a Short Global Dry Eye Symptom Index. The Ocular Surface. 2007;5(1):50-7.

4. Lemp MA. Report of the National Eye Institute/Industry workshop on Clinical Trials in Dry Eyes. Clao J. 1995;21(4):221.

5. Krupin T, Cross DA, Becker B. Decreased Basal Tear Production Associated With General Anesthesia. Arch Ophthalmol. 1977;95:107-8.

6. Craig JP, Nichols KK, Akpek EK, Caffery B, Dua HS, Joo CK, et al. TFOS DEWS II Definition and Classification Report. Ocul Surf. 2017;15(3):276-83.

7. Gukasyan HJ, Kim KJ, Lee V, Kannan R, Glutathione. and Its Transporters in Ocular Surface Defense. 2007;5(4):269-79.

8. Zernii EY, Golovastova MO, Baksheeva VE, Kabanova El, Ishutina IE, Gancharova OS, et al. Alterations in Tear Biochemistry Associated with Postanesthetic Chronic Dry Eye Syndrome. Biochemistry. 2016;81(12):1549-57.

9. Batra YK, Bali IM. Corneal Abrasions During General Anesthesia. Anesthesia Analgesia. 1977;56(3):363. 
10. Hori Y, Spurr-Michaud SJ, Russo CL, Argu“eso P, Gipson IK. Effect of retinoic acid on gene expression in human conjunctival epithelium: secretory phospholipase A2 mediates retinoic acid induction of MUC16. Investigative Ophthalmology \& Visual Science. 2005;46(11).

11. Swami HM, Thakur JS, Bhatia S. Impact of mass supplementation of vitamin A. Indian J Pediatr. 2007;74(5):443-7.

12. Sommer A, Green WR. Goblet Cell Response to Vitamin a Treatment for Corneal Xerophthalmia. Am J Ophthalmol. 1982;94(2):213-5.

13. Alanazi SA, El-Hiti GA, Al-Baloud AA, Alfarhan MI, Al-Shahrani A, Albakri AA, et al. Effects of shortterm oral vitamin A supplementation on the ocular tear film in patients with dry eye. Clinical Ophthalmology (Auckland, NZ). 2019;13.

14. Toshida H, Funaki T, Ono K, Tabuchi N, Watanabe S, Seki T, et al. Efficacy and safety of retinol palmitate ophthalmic solution in the treatment of dry eye: a Japanese Phase II clinical trial. Drug Des Devel Ther. 2017;11:1871-9.

15. Kim EC, Choi JS, Joo CK. A comparison of vitamin a and cyclosporine a $0.05 \%$ eye drops for treatment of dry eye syndrome. Am J Ophthalmol. 2009;147(2):206-13 e3.

16. Sullivan LJ, McCurrach F, Lee S, Taylor HR, Rolando M, Marechal-Courtois C, et al. Efficacy and Safety of $0.3 \%$ Carbomer Gel Compared to Placebo in Patients with Moderate-to-Severe Dry Eye Syndrome. Ophthalmology. 1997;104(9):1402-8.

17. Roth S, Thisted RA, Erickson JP, Black S, Schreider B. Eye injuries after nonocular surgery. A study of 60,965 anesthetics from 1988 to 1992. Anesthesiology. 1996;85(5):1020.

18. Yang S, Guo W, Gong Y, Wang J, Chen L, Zhao J, et al. Application of vitamin A palmitate eye gel and nurse value of Watson's theory of caring in children with dry eye after strabismus surgery: a randomized trial. Transl Pediatr. 2021;10(9):2335-46.

19. Cui X, Xiang J, Zhu W, Wei A, Le Q, Xu J, et al. Vitamin A Palmitate and Carbomer Gel Protects the Conjunctiva of Patients With Long-term Prostaglandin Analogs Application. J Glaucoma. 2016;25(6):487-92.

20. Orlin SE, Kurata FK, Krupin T, Schneider M, Glendrange RR. Ocular lubricants and corneal injury during anesthesia. Anesthesia Analgesia. 1989;69(3):384-5.

\section{Figures}




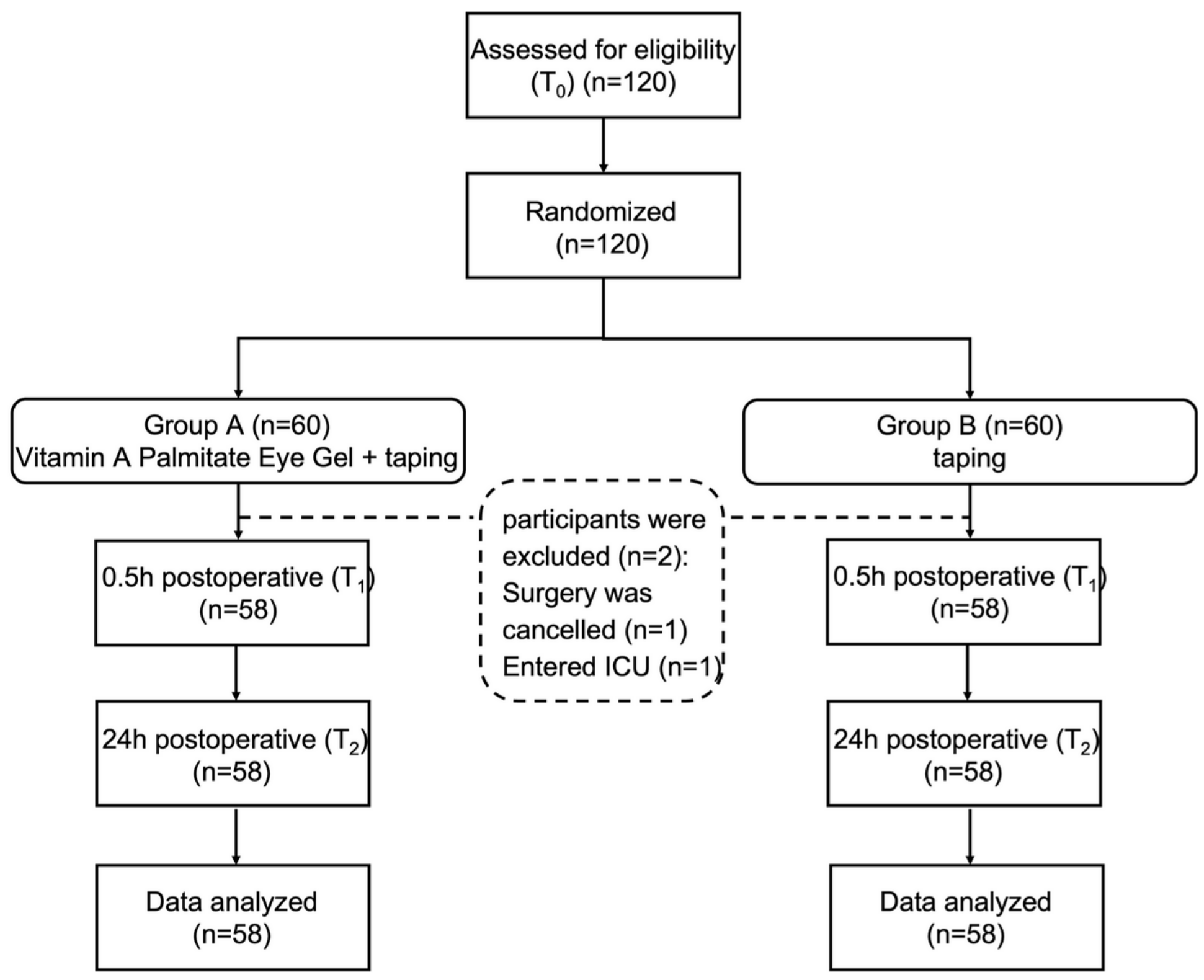

Figure 1

CONSORT flow diagram. 


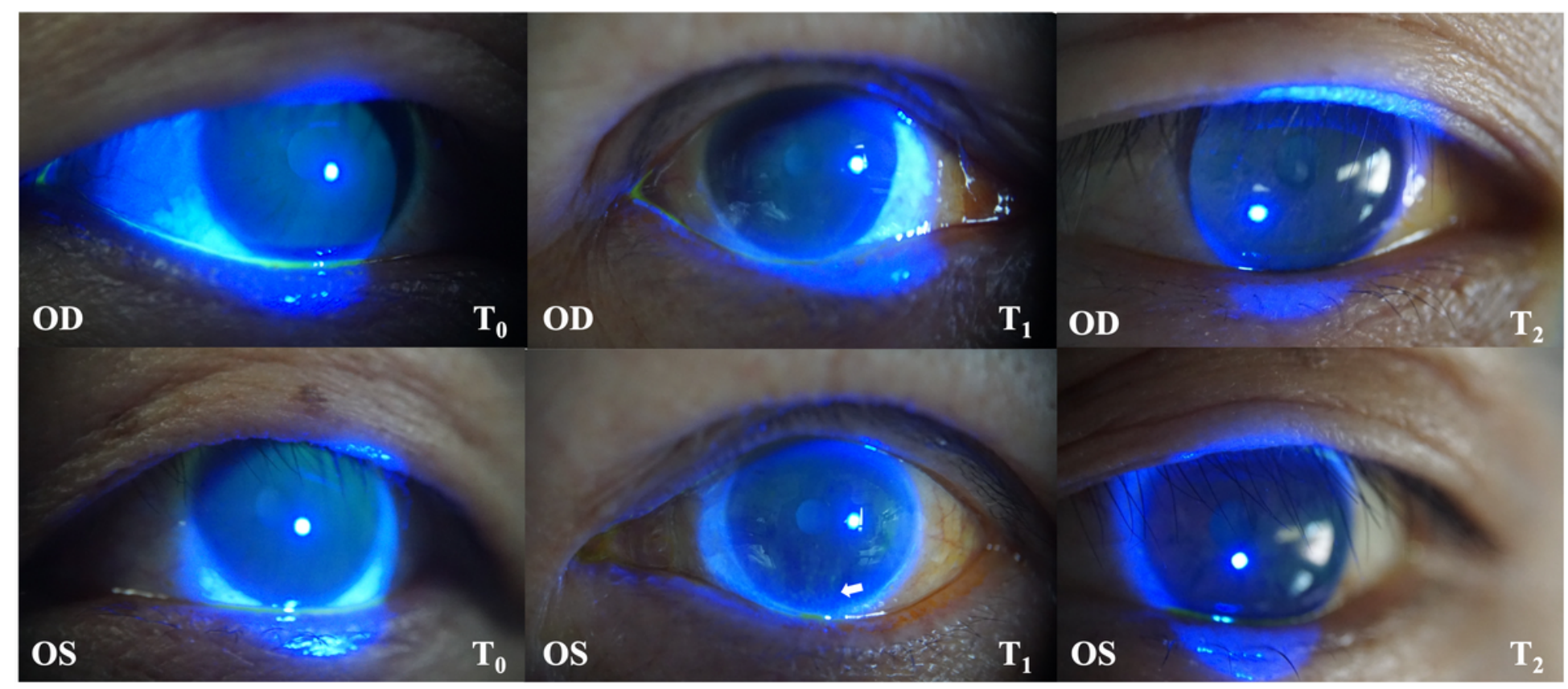

\section{Figure 2}

Hand-held slit lamp corneal fluorescein sodium-stained cobalt blue photography. This participant underwent orthopedic surgery, and the duration of anesthesia was 197 minutes. His conjunctival sac of the right eye (OD) was smeared with VA ophthalmic gel, and the adhesive tape was transversely pasted. His left eye (OS) was simply pasted with adhesive tape. There were several fluorescein dots on the cornea of the left eye at $T_{1}$ (arrow).

\section{Supplementary Files}

This is a list of supplementary files associated with this preprint. Click to download.

- renamed8eafe.doc 